\title{
Combined Aortic Valve Replacement and Coronary Bypass Grafting in Patient With Lipton's R-III Type of Single Coronary Artery Anomaly
}

\author{
Milica Karadzic Kocica ${ }^{1}$, Hristina Ugrinovic ${ }^{1}$, Dejan Lazovic ${ }^{2}$, Nemanja Karamarkovic ${ }^{2}$, Milos Grujic ${ }^{2}$, Borivoje \\ Lukic $^{3}$, Oliver Radmilii ${ }^{3}$, Vladimir Cvetic ${ }^{3}$, Mladen Kocica ${ }^{2, *}$
}

1 Centre for Anesthesiology and Reanimatology, UC Clinical Centre of Serbia, 11000 Belgrade, Serbia; milica.karadzic@gmail.com (M.K.K.); hristinaugrinovic@hotmail.com (H.U.)

2 Clinic for Cardiac Surgery, UC Clinical Centre of Serbia, 11000 Belgrade, Serbia; lazovic.dejan88@gmail.com (D.L.); nemanja_bg89@hotmail.com (N.K.); drgrujicmilos@gmail.com (M.G.); mladen.kocica@kcs.ac.rs (M.K.)

3 Clinic for Vascular and Endovascular Surgery, UC Clinical Centre of Serbia, 11000 Belgrade, Serbia; drboral83@gmail.com (B.L.); oliver_radmili@yahoo.com (O.R.); drvladimrcvetic@gmail.com (V.C.)

* Correspondence: mladen.kocica@kcs.ac.rs; Tel.: +381 (69) 3670-609

Citation: Lastname, F.; Lastname, F.; Lastname, F. Title. J. Cardiovasc. Dev. Dis. 2021, 8, x.

https://doi.org/10.3390/xxxxx

\begin{abstract}
A single coronary artery is a very rare condition, commonly associated with other congenital anomalies. It could be generally classified as neither benign nor malignant form of congenital coronary artery anomalies since its pathophysiological and clinical implications grossly depend on different anatomical patterns defined by the site of origin and distribution of the branches. By presenting the patient with an isolated single coronary artery, who underwent successful combined aortic valve replacement and coronary artery bypass grafting surgery, we intend to distinguish casual from causal in this extremely rare clinical and surgical scenario. This is the first-ever case published, combining such underlying pathology, clinical presentation, and surgical treatment.
\end{abstract}

Keywords: single coronary artery; aortic valve surgery, coronary artery bypass grafting surgery; case report;

\section{Introduction}

The first anatomical description of a single coronary artery (SCA) was published in 1716 by Adam Christian Thebesius [1] and the first antemortem diagnosis of this congenital coronary artery anomaly (CCAA) was independently reported in 1967, by Halperin and Toshima. [2,3]

By definition, SCA is CCAA with anomalous coronary artery (CA) connection to the aorta/systemic circulation in which all of the coronary blood flow is provided by an artery that arises by one ostium from an arterial trunk. [4, 5]

Although the very rare condition, accounting for less than $3 \%$ of all CCAA, affecting $0.0024 \%-0.066 \%$ of those undergoing coronary angiography and $0.019 \%-0.4 \%$ of the general population, SCA carries both natural and/or procedural risks that may affect normal life expectancy. This neither benign nor malignant form of CCAA may cause sudden cardiac death in $23 \%$ of carriers. In more than $40 \%$ of the reported cases, SCA is associated with other complex congenital anomalies (e.g. transposition of the great arteries, tetralogy of Fallot, truncus arteriosus, coronary arteriovenous fistula, endocardial fibroelastosis or bicuspid aortic valve). [5-8]

Here we present a patient with both risks linked to an existent Lipton's R-III type of isolated SCA, [9] who underwent successful combined aortic valve replacement (AVR) 
and coronary artery bypass grafting (CABG) surgery. This is the first-ever published case with such preoperative and surgical characteristics, and together with a very few similar reported cases, would hopefully improve our understanding of "casual and causal" in SCA's clinical presentation. [10]

\section{Case Report}

\subsection{Preoperative Evaluation}

A 55 years-old $(176 \mathrm{~cm}, 92 \mathrm{~kg})$ male patient was admitted for scheduled combined AVR and CABG surgery. Except for hyperlipidemia, smoking, and heredity, no other risk factors for atherosclerosis were documented. No history of previous myocardial infarctions (MI), chronic diseases, and hospitalizations. Screening color Doppler of the extracranial brain blood vessels found the chronic asymptomatic occlusion of the right internal carotid artery and 30\% stenosis at the opposite side. De novo exertional angina appeared two months before the admission so that he underwent a complete cardiological examination. Prehospital therapy included: Trimetazidine 2x35mg, Metoprolol 2x25mg, Rosuvastatin 1x10mg, Aspirine 1x100mg, and Clopidogrel 1x75mg, On repeated electrocardiographic (ECG) traces, a sinus rhythm, with $65-70$ beats/min, with rS and negative T in D3 and aVF was recorded. Cardiac exercise stress testing revealed decreased coronary blood flow reserve. Trans-thoracic echocardiography (TT-ECHO) indicated the presence of moderate trileaflet aortic valve (AoV) stenosis (Area $1.2 \mathrm{~cm}^{2}$, MPG $19 \mathrm{mmHg}$, Velocity $3.2 \mathrm{~m} / \mathrm{s}$ ) and mild regurgitation, with preserved left ventricular kinetics, morphology, and function (LV-EDD $5.3 \mathrm{~cm}$, LV-ESD $3.3 \mathrm{~cm}, \mathrm{~S}$ and PV $0.9 \mathrm{~cm}$, EF 65\%, FS 35\%). Subsequent trans-radial selective coronary angiography (Figure 1) revealed an SCA anomaly arising from the right sinus of Valsalva. Triple vessel CA disease was present, with proximal occlusion of dominant right coronary artery (RCA), distal 70\% - 90\% stenosis of retroaortic circumflex artery (RCx), and mid-to-distal 50\% stenosis of left anterior descending (LAD) artery. Distal RCA branches had good retrograde filling from the LAD.
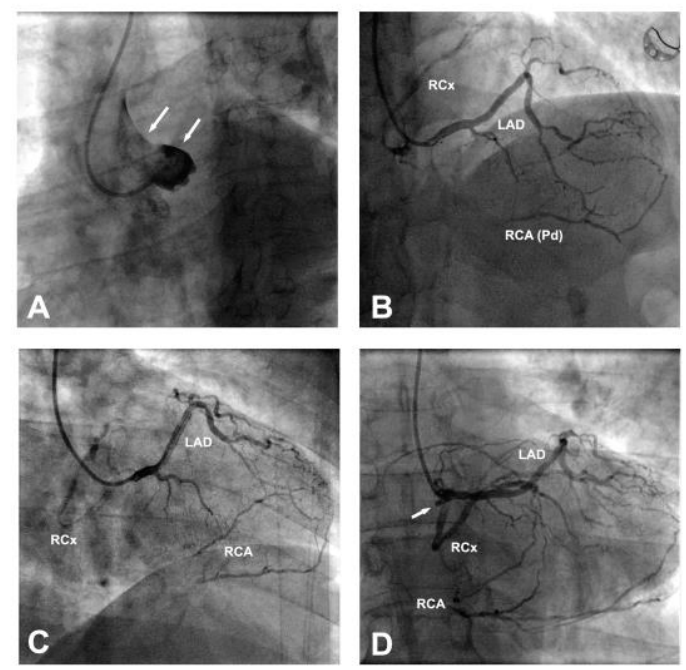

Figure 1. - Selective coronary angiography: A - Absence of any CA (arrows) from the left sinus of Valsalva (LAO: 37, Caudal: 32); B - SCA (Lipton's R-III type) from the right sinus of Valsalva, with anterior LAD course, retroaortic RCx course with distal stenosis (70-90\%) and proximally occluded RCA with retrograde Pd filling from LAD (RAO: 2, Cranial: 41); C - Same as B but adjusted for better LAD and RCx tracing (RAO: 31, Cranial: 5); D - Same as B adjusted for the proximal RCA stump (arrow) tracing (LAO: 3, Caudal: 2). Legend: LAO - Left anterior oblique; RAO - Right anterior oblique; LAD - Left anterior descending; RCx - Circumflex; RCA - Right coronary artery. 
For better spatial orientation, multidetector computed tomography (MDCT) coronary angiography was performed and subsequent 3-D coronary network reconstruction was done (Figure 2). This confirmed the retroaortic RCx course and its tight proximity to the aortic root, a fact of great importance for the forthcoming surgical procedure. Also, the anterior course of the LAD was evident, being a better option for future hemodynamics. Thus, it was possible to classify this SCA anatomy into Lipton's R-III type. The patient was prepared and referred for combined AVR and CABG surgery.

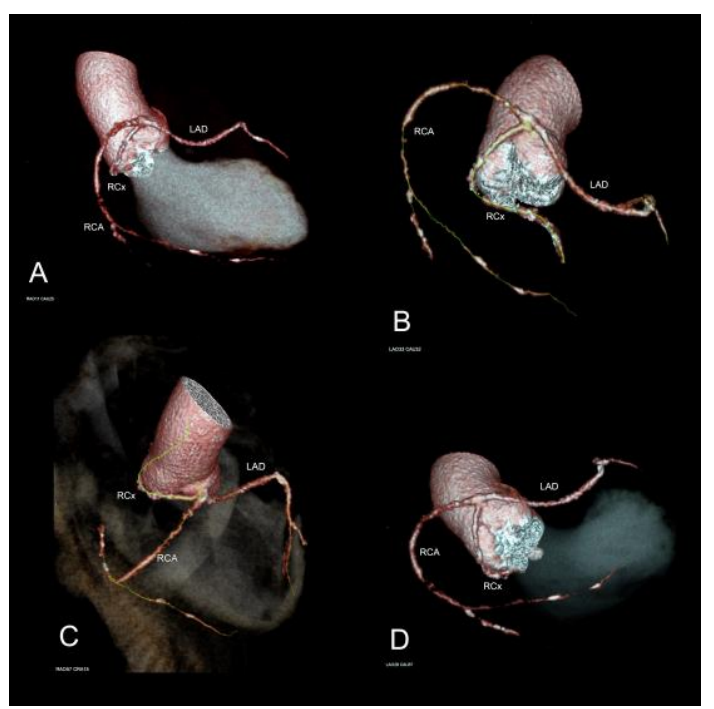

Figure 2. - Multidetector computed tomography (MDCT) 3-D coronary angiography: Different angles analysis to get a clear insight in distal branching patterns and particularly in retroaortic $\mathrm{RCx}$ course in respect to the aortic root. A - RAO: 11, Caudal: 25; B - LAO: 33, Caudal: 32; C - RAO: 57, Cranial: 15; D - LAO: 28, Caudal: 37. Legend: Same as Figure 1.

\subsection{Surgical Treatment}

Standard median sternotomy and heart exposure was performed and no apparent macroscopical evidence of any congenital heart malformation was present. Mid-to-distal portions of RCA, RCx, and LAD were easily identified at their usual locations. Partial cardiopulmonary bypass was established, left atrium vented via the right superior pulmonary vein and aortic cross-clamp placed. The heart was arrested with cold St.Thomas cardioplegic solution. Transverse aortotomy exposed tricuspid AoV with degenerative nodular fibro-calcified changes predominantly at leaflets' base, without any fusion of the cusps confirmed by surgical inspection. The free edge of the right leaflet was elongated with the cleft-like formation, probably caused by coalescing and subsequent rupture of the para-commissural fenestrations. There were no coronary ostia in the left sinus of Valsalva (Figure 3).

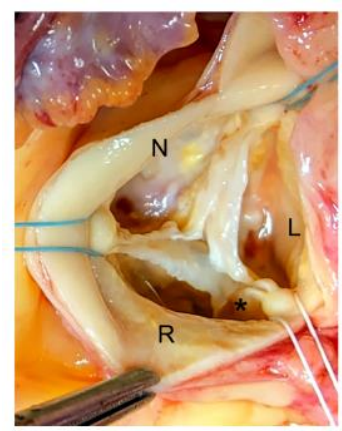

A

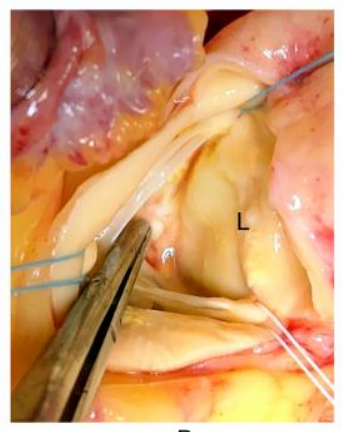

B 
Figure 3. - Aortic valve and Valsalva sinuses - intraoperatively: A - Trileaflet AoV, with degenerative nodular fibro-calcified changes predominantly at leaflets' base. Elongation of the free edge of the $\mathrm{R}$ leaflet with para-commissural cleft-like formation (asterisk); B - The absence of coronary ostium in the left sinus of Valsalva. Legend: L - left; R - right; N - non-coronary.

The single, wide para-commissural ostium of the common mixed trunk [4] with slightly dilated periostial aortic wall, forming a kind of secondary sinus, was identified within the right sinus of Valsalva (Figure 4).

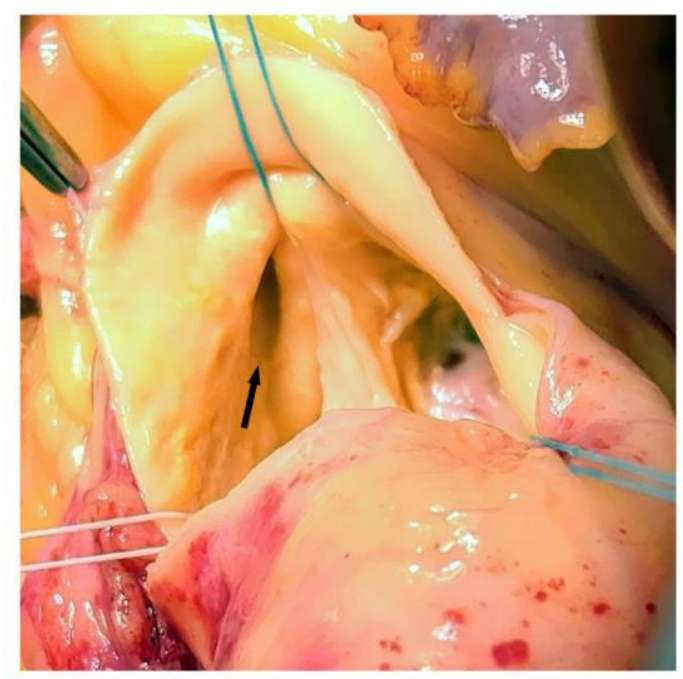

Figure 4. - SCA ostium - intraoperatively: Wide para-commissural ostium of the common mixed trunk (arrow) [4], arising from the R sinus of Valsalva. Periostial aortic wall is slightly dilated, forming a kind of secondary sinus within the R sinus of Valsalva (see also Figure 2-C)

Valvular excision and meticulous decalcification were done so that a $21 \mathrm{~mm}$ St. Jude Medical $^{\mathrm{TM}}$ Regent $^{\mathrm{TM}}$ (St. Jude Medical Inc., St. Paul, MN, USA) mechanical aortic prosthesis could be easily implanted. We decided to choose smaller (i.e. $21 \mathrm{~mm}$ ) of two prosthetic sizes (i.e. 21 and $23 \mathrm{~mm}$ ) with effective orifice area index greater than $1 \mathrm{~cm}^{2} / \mathrm{m}^{2}$ to avoid a "tight fit" scenario and subsequent retroaortic RCx compression. Having completed the distal anastomoses on RCx (OM1) and RCA (Pd) with saphenous vein grafts, the aortic valve prosthesis was sewn in place. Extreme care was taken not to go too deep with the stitches, to avoid the inadvertent injury of the retroaortic RCx. To ensure equal distribution during repeated cardioplegia delivery, we used the widest direct coronary cannula with a silicone cuff, to fit the single ostium. The next concern, after the aortotomy was closed and proximal anastomoses were sewn in place, was to precisely identify the LAD course over the RV cone, to avoid its injury during the epicardial pacing wire implantation. The entire procedure was uneventful and the patient was easily weaned from cardiopulmonary bypass.

\section{Discussion}

The combination of underlying pathology, clinical presentation, and surgical treatment in the case we presented here is extremely rare. Having performed an extensive literature review, we could not find any case report of an adult patient with isolated SCA, AoV stenosis, and CA disease, submitted to combined AVR and CABG surgery. Katsetos and associates have reported a similar case in 2003, but in a patient with AoV insufficiency. [11] In a similar report by Gallo and associates and Kawano and associates, AVR was done without CABG. $[12,13]$ In four other cases, AVR was done in patients with SCA and associated congenital anomalies. [11, 12]

It has been well recognized by the Development, Anatomy and Pathology Working Group of the European Society of Cardiology, that the continuous path 
from-bench-to-bedside is to be followed to understand and treat CCAA. [5] Embryological development of proximal CA and their orifices has been a matter of debate. [5, 14-17] Cellular and molecular mechanisms, governing the connection of coronary and systemic circulation during the embryogenesis, include selective and "controlled aortic wall invasion", by sprouts from the peritruncal subepicardial capillary plexus (ring). (17) Why do peritruncal sprouts selectively invade aortic and not pulmonary root, and why do they prefer aortic sinuses of Valsalva adjacent to the pulmonary trunk, remains unclear. The cardiac neural crest, although without any cellular contribution to the coronary blood vessels, has been shown to play an important regulatory role in this process. Surgical ablation of cardiac neural crest cells resulted in different anomalous origins and courses of CA and was also associated with aortic root malformations (e.g. bicuspid AoV) and patterning anomalies of great arteries. Along with neural crest contribution, proximal CA stems and their aortic orifices are finally established by series of apoptotic events and mural recruitments (pericytes, smooth muscle cells), governed by the complex interplay of different families of growth and transcription factors. [5, 14-17] These embryological blueprints connecting the aortic root and proximal CA, deserve an effort to distinguish casual from causal in our case.

Clinical or morphologic evidence of myocardial ischemia, as a natural risk, is present in about $15 \%$ of isolated SCA cases, without any angiographic evidence of atherosclerotic CA disease. On the other hand, $40 \%-45 \%$ of isolated SCA cases have significant atherosclerotic narrowing of one or more major epicardial CA. [18] Rigatelli and associates consider that benign forms of CCAA, in the absence of significant known risk factors, do not accelerate CA atherosclerosis. [19] Accordingly, the isolated SCA in our case is probably just a casual finding in present CA atherosclerosis, but we could not completely exclude, at least, its contributing role. Single wide ostium, different branching patterns with unusual angulations, the intrinsic inability to develop proximal collateral branches, and altered relations with surrounding anatomical structures may be predisposing factors for the site-specific plaque formation, due to altered blood rheology and specific coronary wall stress distribution. [20-23] If not related to the etiopathogenesis, these congenital anatomical features may be a reason for different reactivity of the CA network and, thus, for the clinical presentation. Lo Rito and associates have found that RCA with anomalous aortic origin fails to expand its lumen and increase flow at incremental loading conditions. They used structural finite element analysis simulations of pressure increasing in the aortic root, and found the absence of dilation in the intramural portion of the RCA, suggesting that this may be the anatomic substrate responsible for the reduction of coronary blood flow reserve, regardless of inability to demonstrate inducible ischemia on the exercise stress testing. [24] A further patient-specific computational model simulation framework is needed to see whether their results also stand for isolated SCA. Accordingly, it seems reasonable to suppose that our patient would have a different clinical presentation of CA atherosclerotic diseases in the absence of SCA. The procedural risks defined as diagnostical, interventional, and surgical pitfalls and hazards related to the presence of SCA are also very important in a particular case. In a case with combined AVR and CABG, we previously described, having anomalous aortic origin (i.e. high take-off) of RCA and RCx, we emphasized the importance of both natural and procedural risks. [6] Although invasive coronary angiography is the gold standard for SCA and CA disease detection, MDCT coronary angiogram is necessary to precisely delineate the origin, branching patterns, and the course of the anomalous arteries and thus has an important role in distinguishing between different types of SCA. [25] In our case (Figure 2), MDCT revealed three important SCA features: 1) very short common mixed trunk - bearing possible difficulties during the direct cardioplegia delivery, 2) anterior LAD course - being prognostically better than interarterial course but in the risk of injury during the epicardial pacing, and 3) retroaortic RCx course running very close to the aortic root and in the risk of injury during and after AoV implantation. With the possibility to know all these features preoperatively, it was feasible 
to anticipate all pitfalls and hazards, and perform the complex surgical procedure safely and unenventfully.

Beyond SCA and CA disease, the third part of the complex underlying pathology, in this case, was the AoV stenosis. In all similar cases reported, functional AoV lesion was valvular insufficiency. [11-13] While Katsetos and associates could not find any causal relation between SCA and AoV insufficiency, [11] Kawano and associates speculated that AoV deformity likely resulted from adaptive modification of a congenitally malformed aortic valve, based on histologic and immunohistochemical leaflet properties. [12] Although in a series of 1099 consecutive patients, submitted to AoV surgery, of whom $4 \%$ with CCAA, Naito and associates could not find any SCA. They believe that younger patients, who were referred for AoV surgery, had a certain congenital component in their AoV disease, leading towards a higher prevalence of anomalous coronary ostium, thus causally linking these two pathologies. They also could not find any significant correlation between CCAA and functional AoV lesions, but they did so for the root-type aortopathy. [26] As the risk factors for atherosclerosis and degenerative disease of the trileaflet aortic valve are similar, [27] it is reasonable to consider that the presence of SCA in our case is only a casual finding. The presence of advanced carotid and CA artery disease could only strengthen this conclusion. However, we should not ignore a fact that anomalous origins of CA could be associated with aortic root pathology. Accordingly, a slightly dilated periostial aortic wall, forming a kind of secondary sinus within the right sinus of Valsalva is probably congenital (see Figures 4 and 2-C). Its presence may subsequently induce adaptive modification and degenerative changes of the $\mathrm{AoV}$, by changing the stress loading of the aortic valve leaflets. $[12,27,28]$ Thus, again, the line between casual and causal becomes obscure.

\section{Conclusions}

Isolated SCA is an extremely rare, neither benign nor malignant form of CCAA, carrying a natural risk for sudden cardiac death of $23 \%$. Casually and/or causally associated with CA and/or AoV diseases, it brings along additional procedural risks. As for all other CCAA, both invasive and MDCT coronary angiography is mandatory for the patients with isolated SCA. By presenting this extremely rare case with Lipton's R-III type of isolated SCA, who underwent successful combined AVR and CABG surgery, we emphasized the importance of meticulous, case-specific preoperative "bench-to-bedside" analysis, to ensure the optimal outcome.

Author Contributions: Conceptualization: M.K.K., M.K.; Methodology: M.K.K., H.U., D.L., N.K., M.G.; Validation: M.K.K., M.K.; Formal analysis: M.K.K., H.U. D.L., N.K., M.G., B.L., O.R., V.C.; Investigation: M.K.K., H.U., D.L., N.K., M.G., B.L., O.R., V.C.; Data curation: H.U., D.L., N.K., M.G, B.L., O.R., V.C.; Writing: M.K.K., M.K.; Visualization: B.L., O.R., V.C.; Supervision: M.K.; All authors have read and agreed to the published version of the manuscript.

Funding: This research received no external funding

\section{Ethical Statement}

The study was conducted according to the guidelines of the Declaration of Helsinki. As all figures used contain no private health information and there is no identification possible, Institutional Review Board approval was not necessary. The manuscript was prepared with consent from the patient.

Conflicts of Interest: The authors declare no conflict of interest.

\section{References}

1. Smith JC. Review of single coronary artery with report of 2 cases. Circulation. 1950;1(5):1168-75. DOI:10.1161/01.cir.1.5.1168

2. Halperin IC, Penny JL, Kennedy RJ. Single coronary artery. Antemortem diagnosis in a patient with congestive heart failure.

Am J Cardiol. 1967;19(3):424-7. DOI:10.1016/0002-9149(67)90456-0. 
3. Toshima H, Sasaki K, Oshima F, Tanaka K, Fukami T. Single Coronary Artery. Case Report. Kurume Med J. 1967;14(3):89-93. DOI:10.2739/kurumemedj.14.89

4. Angelini P, Villason S, Chan AV, Diez JG. Normal and Anomalous Coronary Arteries in Humans. In: Angelini P, Fairchild VD, editors. Coronary Artery Anomalies: A Comprehensive Approach: Lippincott Williams \& Wilkins; 1999. p. 27-79.

5. Pérez-Pomares JM, de la Pompa JL, Franco D, Henderson D, Ho SY, Houyel L, et al. Congenital coronary artery anomalies: a bridge from embryology to anatomy and pathophysiology--a position statement of the development, anatomy, and pathology ESC Working Group. Cardiovasc Res. 2016;109(2):204-16. DOI:10.1093/cvr/cvv251.

6. Kočica M, Karadžić M, Grujić M, Cvetković D, Šoškić L. Anomalous aortic origin of right and circumflex coronary arteries procedural risks during combined aortic valve replacement and coronary artery bypass grafting. Srp Arh Celok Lek. 2018;146(7-8):440-4. DOI:10.2298/SARH180417047K

7. Mavroudis C, Dodge-Khatami A, Stewart RD, Jacobs ML, Backer CL, Lorber RE. An overview of surgery options for congenital coronary artery anomalies. Future Cardiol. 2010;6(5):627-45. DOI:10.2217/fca.10.82.

8. Shah JR, Priya C, Om T. Single coronary artery: Classification and MDCTA diagnosis. Eur J Radiol. 2011;77(1):e1-e4. DOI:10.1016/j.ejrex.2010.10.007

9. Lipton MJ, Barry WH, Obrez I, Silverman JF, Wexler L. Isolated single coronary artery: diagnosis, angiographic classification, and clinical significance. Radiology. 1979;130(1):39-47. DOI:10.1148/130.1.39

10. Angelini P. A casual versus causal relationship in coronary artery anomalies :a question of method. Tex Heart Inst J. 2004;31(3):276-7. PMCID:521771

11. Katsetos MC, Toce DT. Single coronary artery with aortic regurgitation. Cardiovasc Intervent Radiol. 2003;26(6):567-8. DOI:10.1007/s00270-003-2736-4

12. Kawano H, Hayashi T, Minami T, Koide Y, Eishi K, Maemura K. Cleft-like formation of the aortic valve in an adult patient with a single coronary artery. Intern Med. 2012;51(17):2341-5. DOI:10.2169/internalmedicine.51.7808

13. Gallo M, Lika A, Gerosa G, Colli A. Aortic valve replacement in a single coronary artery arising from the right Valsalva sinus. Eur J Cardiothorac Surg. 2013;43(5):e141. DOI:10.1093/ejcts/ezt020

14. Angelini P, Velasco JA, Flamm S. Coronary anomalies: incidence, pathophysiology, and clinical relevance. Circulation. 2002;105(20):2449-54. DOI:10.1161/01.cir.0000016175.49835.57

15. Tomanek RJ. Formation of the coronary vasculature: a brief review. Cardiovasc Res. 1996;31 Spec No:E46-51. PMID: 8681345

16. von Kodolitsch Y, Ito WD, Franzen O, Lund GK, Koschyk DH, Meinertz T. Coronary artery anomalies. Part I: Recent insights from molecular embryology. Z Kardiol. 2004;93(12):929-37. DOI:10.1007/s00392-004-0152-7

17. Bernanke DH, Velkey JM. Development of the coronary blood supply: changing concepts and current ideas. Anat Rec. 2002;269(4):198-208. DOI:10.1002/ar.10139

18. Shirani J, Roberts WC. Solitary coronary ostium in the aorta in the absence of other major congenital cardiovascular anomalies. J Am Coll Cardiol. 1993;21(1):137-43. DOI:10.1016/0735-1097(93)90728-j

19. Rigatelli G, Gemelli M, Zamboni A, Docali G, Rossi P, Rossi D, et al. Are coronary artery anomalies an accelerating factor for coronary atherosclerosis development? Angiology. 2004;55(1):29-35. DOI:10.1177/000331970405500105

20. Villa AD, Sammut E, Nair A, Rajani R, Bonamini R, Chiribiri A. Coronary artery anomalies overview: The normal and the abnormal. World J Radiol. 2016;8(6):537-55. DOI:10.4329/wjr.v8.i6.537

21. Madan N, Lorber R, Nento D, Mumtaz M, Singh HR. Is Interarterial Course a Requisite for Ischemia in Anomalous Origin of Coronary Arteries? World J Pediatr Congenit Heart Surg. 2017;8(4):533-6. DOI:10.1177/2150135116644698

22. Eyüboğlu M, Cüce F. Single coronary artery accompanying myocardial bridging on LAD and retroaortic course of LCX. Int J Cardiovasc Acad. 2015;1(2):53-5. DOI:10.1016/j.ijcac.2015.09.001

23. Kaleda VI. R-IIIC type: non-simple single coronary artery. Eur J Cardiothorac Surg. 2014;45(1):207. DOI:10.1093/ejcts/ezt337 
24. Lo Rito M, Romarowski RM, Rosato A, Pica S, Secchi F, Giamberti A, et al. Anomalous aortic origin of coronary artery biomechanical modeling: Toward clinical application. J Thorac Cardiovasc Surg. 2020. DOI:10.1016/j.jtcvs.2020.06.150

25. Al Umairi R, Al-Khouri M. Prevalence, Spectrum, and Outcomes of Single Coronary Artery Detected on Coronary Computed Tomography Angiography (CCTA). Radiol Res Pract. 2019;2019:2940148. DOI:10.1155/2019/2940148

26. Naito S, Petersen J, Reichenspurner H, Girdauskas E. The impact of coronary anomalies on the outcome in aortic valve surgery: comparison of bicuspid aortic valve versus tricuspid aortic valve morphotype. Interact Cardiovasc Thorac Surg. 2018;26(4):617-22. DOI:10.1093/icvts/ivx396

27. Robicsek F, Thubrikar MJ, Fokin AA. Cause of degenerative disease of the trileaflet aortic valve: review of subject and presentation of a new theory. Ann Thorac Surg. 2002;73(4):1346-54. DOI:10.1016/s0003-4975(01)03001-6

28. Robicsek F, Thubrikar MJ. Role of sinus wall compliance in aortic leaflet function. Am J Cardiol. 1999;84(8):944-6, a7. DOI:10.1016/s0002-9149(99)00475-0 